\title{
23\% Efficiency Metal Wrap Through Silicon Heterojunction Solar Cells
}

\author{
G. Coletti ${ }^{1}$, F. Ishimura ${ }^{2}$, Y. Wu ${ }^{1}$, E.E. Bende ${ }^{1}$, G.J.M. Janssen ${ }^{1}$, B.B. van Aken ${ }^{1}$, K.Hashimoto ${ }^{2}$, Y.Watabe ${ }^{2}$ \\ ${ }^{1}$ ECN Solar Energy, Petten, NL-1755 LE, the Netherlands, tel. +31 88 5154382, e-mail: coletti@ecn.nl \\ ${ }^{2}$ Choshu Industry Co., Ltd., 3740, Shin-yamanoi, Sanyo Onoda, Yamaguchi 757-8511, Japan
}

\begin{abstract}
MWT-SHJ cells and modules combine the positive benefits of both underlying technologies: namely high $V_{\text {oc }}$, higher $J_{\text {sc }}$ and higher FF. Especially the FF is maintained before and after encapsulation thanks to the rear interconnection which strongly reduces cell to module FF losses. We obtained two record efficiencies for these devices with two different front metallizations: $22.6 \%$ using low temperature Ag paste and $23.1 \%$ using copper plating. $V_{o c}$ values above $730 \mathrm{mV}$ have been achieved also in the new $6 \times 6$ vias metallization, demonstrating that the architecture maintains the exceptional passivation typical of heterojunction devices. The MWT cell and module structure offers even greater advantages on heterojunction solar cells: i) front side Ag consumption reduction up to a factor two; ii) concurrent low temperature cell interconnection and encapsulation. Our record MWT-SHJ solar cells and modules are manufactured using industrially proven tools and 6 inch commercial n-type $\mathrm{Cz}$ Si wafers. The metallization choice gives ample room to manufacturers for optimization based on internal cost structure, material costs and business strategy. MWT-SHJ behavior at reverse bias voltage and low illumination intensity is comparable to conventional $\mathrm{HJ}$ devices.
\end{abstract}

\section{INTRODUCTION}

High efficiencies and low materials consumption are the main drivers towards low-cost $(\$ / \mathrm{Wp})$ silicon PV modules.

Several solar cell technologies have demonstrated the ability to reach $22 \%$ cell efficiency. In addition, new back contact module technologies are now available to overcome losses caused by the interconnection and due to the limited width of the tabs.

In this contribution we show the most recent development in the successful combination of Metal Wrap Through (MWT) architecture with Silicon Heterojunction (SHJ) solar cells.

The vast majority of the market implements module technologies based on interconnection of solar cells in strings by tabs soldered from the front of one cell to the rear of the adjacent one. To limit shading losses caused by these tabs, such interconnection leads to additional resistive losses in a string of cells thereby reducing the module performance. MWT technology provides a relatively small step from conventional cell technologies and has already demonstrated to increase the module power by $3 \%$, and up to at least $5 \%$ is anticipated [1]. This is possible thanks to an integrated cell and module design in which conductive interconnection foil is used to eliminate cell-to-module power loss compared to conventional tabbing technology. Part of this gain is thanks to the reduced metal coverage on the front side, giving the solar cell performance a potential efficiency increase up to about
2.5\% relative. Furthermore, thanks to the unit cell concept (the front contact of each unit cell connected to a rear contact by a through-cell via), MWT cell structure decouples the wafer size from metallization requirements allowing for better cell-to-module power ratio.

Front and rear contacts heterojunction solar cells have demonstrated more than $24 \%$ cell efficiency $(25.6 \%$ on interdigitated back contact [3]) achieving excellent surface passivation with Voc exceeding $730 \mathrm{mV}$ [2]. Furthermore SHJ has a lower temperature coefficient, resulting in higher module energy yield under real operating conditions.

Nevertheless the low temperature Ag paste required for contact formation in SHJ devices is still today a challenge as it suffers from high cost and low conductivity resulting in high Ag consumption. Solutions such as multi-wire interconnection and multi-busbars have been introduced to tackle this conductivity issue [4,5].

MWT-SHJ architecture provides an elegant solution to high cost and reduced conductivity of low temperature silver pastes while maintaining the above mentioned advantages of SHJ.

Moreover, the low-temperature process required by heterojunction is perfectly met by the soldering-free MWT module technology which uses conductive adhesive and single step curing for interconnection and encapsulation.

MWT and SHJ cell technologies are also compatible with next generation thinner wafers resulting in a win-win situation both on cell and module level.

MWT-SHJ devices combine all the advantages of the individual concepts in a device with high open circuit voltage, high short circuit current and high module power thanks to reduced power loss.

In this paper, we show the most recent results of this MWTSHJ solar cell architecture which resulted in a record efficiency of 23\% total area using commercial n-type $\mathrm{Cz} 6$ inch wafers.

\section{PURPOSE OF THE WORK AND APPROACH}

In this contribution we describe recent development of metal wrap through (MWT) silicon heterojunction (SHJ) solar cells and modules. We used a rear emitter cell structure to reduce recombination and shunt at the vias as previously reported [6]. Our approach is both theoretical and experimental. We used a 2D model to predict the experimental results and chose the best configuration and geometry of the MWT unit cells. The model has been then validated by 
fabricating real working devices and characterization (IVcurve and EL).

In addition here we demonstrate this approach with both low temperature Ag paste screen printing and $\mathrm{Cu}$ plating.

\section{SCIENTIFIC INNOVATION AND RELEVANCE}

To our knowledge, the solar cell performances obtained are record efficiency for both MWT-SHJ and MWT devices. The use of conventional low temperature $\mathrm{Ag}$ paste and low temperature interconnection methods (i.e. conductive tape, multi wire etc.) provide a costly solution that hinder the entrance in mass production of Si heterojunction technology. MWT-SHJ addresses both issues. The MWT structure allows the reduction of the current to be transported thanks to the reduction of the unit cell size at virtually no-cost and without the complicated hassle of upscaling to multi-busbar or multiwire (high T) or smart-wire (low T) technologies. This allows a reduction of front $\mathrm{Ag} \mathrm{CoO}$ up to a factor two accounting both the front side metallization and interconnection. We expect a similar gain from the rear metallization. On module level, the cells are interconnected through the conductive rear side foil by means of conductive adhesives. During lamination, interconnection takes place at low temperature concurrently to encapsulation.

\section{CELL STRUCTURE}

MWT solar cells have the same architecture of a conventional solar cell with the addition that the front metal contact is wrapped through the wafer through metallized via holes, providing both emitter and base contacts on the rear side (Fig. 1) [6]. As the cell interconnection does not require tabbing and MWT unit cell can be reduced independently from wafer size, the busbar are significantly slimmed down enhancing the light harvesting in comparison to conventional H-pattern cells.

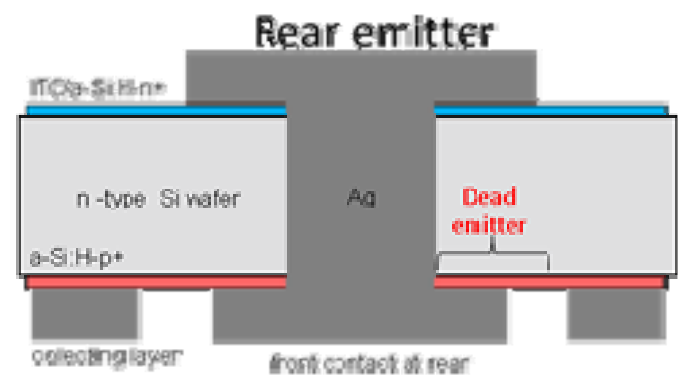

Figure 1. Structure of a rear emitter MWT-SHJ solar cell. The diagram is not in scale. The collecting layer represents both the TCO and Ag rear contact.

\section{EXPERIMENT AND RESULTS}

6" industrial n-type $\mathrm{Cz}$ monocrystalline full-square silicon wafers about $180 \mu \mathrm{m}$ thick were used as substrate for MWTSHJ solar cell fabrication. Conventional heterojunction processing sequences were used for the fabrication of the solar cells: texturization and cleaning, a-Si:H deposition by PECVD and TCO deposition. The MWT-SHJ solar cell process is the same as the SHJ process with adding a few extra steps typical for MWT cell technology [1]: vias drilling, via filling and isolation of the two metal contacts at the rear. In this experiment, the vias drilling is done before the surface preparation for the a-Si:H depositions, therefore the damage removal is done without adding any extra chemical step. In the MWT-SHJ process used, an isolation of the metal plug from the underlying $\mathrm{Si}$ or a-Si:H layer is not necessary. The via filling is done within the same printing step of the rear side pad.

Two types of metallization have been used: i) low temperature silver paste by screen printing and ii) Copper plating of the front metallization. The copper plating has been entirely developed by CIC. A description of this process is described in a sister publication [7].

The rear side of both devices is identical and it can be carried out in two different ways: i) by screen printing and ii) by PVD (Ag blanket).

Table I. IV-results of the MWT-SHJ solar cells. Cell area $243 \mathrm{~cm}^{2}$. Total area efficiency

\begin{tabular}{|l|c|c|c|c|c|}
\hline Structure & $\begin{array}{c}\text { Front } \\
\text { metal }\end{array}$ & $\begin{array}{c}\text { Jsc } \\
\left(\mathbf{m A} / \mathbf{c m}^{2}\right)\end{array}$ & $\begin{array}{c}\text { Voc } \\
(\mathbf{m V})\end{array}$ & $\begin{array}{c}\text { FF } \\
(\mathbf{\%})\end{array}$ & $\begin{array}{c}\text { Eta } \\
(\mathbf{\%})\end{array}$ \\
\hline H-pattern & $\mathrm{Ag} \mathrm{3BB}$ & 38.9 & 722 & 77.3 & 21.7 \\
\hline MWT 4x4 & $\mathrm{Ag}$ & 39.1 & 726 & 76.6 & 21.7 \\
\hline MWT 6x6 & $\mathrm{Ag}$ & 39.2 & 723 & 77.6 & 22.0 \\
\hline MWT 6x6 & $\mathrm{Ag}$ & 39.3 & 719 & 80.0 & $\mathbf{2 2 . 6}$ \\
\hline
\end{tabular}

In table I and Fig. 2, all the cells are measured at ECN according to the ASTM-E948 norm using a Wacom class AAA solar simulator and spectral mismatch correction. Solar cell efficiency is total area.

The first three devices in Table I are manufactured in the same batch to compare the different configuration (front and rear contacts, MWT with $4 \times 4$ vias and MWT with $6 \times 6$ vias). Only the busbar width and number have been changed from configuration to configuration as in MWT the current transport is carried out in the rear side. The number and width of fingers have been kept constant (same finger metal coverage). This allowed making a direct comparison among the different configuration. The total front metal shading for the three devices is $5.6 \%, 3.7 \%$ and $3.4 \%$ for the $3 \mathrm{BB}$, MWT 
4x4 and MWT 6x6, respectively. The last development carried out with LT-Ag metallization $\left(4^{\text {th }}\right.$ cell in Tab I) consists in reducing the rear dead emitter area by about $30 \%$ as suggested in [6]. The dead emitter area reduction is estimated to improve the efficiency by $0.2 \%$ (mostly due to $\mathrm{FF}$ ). At the same time the a-Si deposition was improved resulting in an efficiency gain of $0.6 \%$.

We estimated that with $6 \times 6$ configuration and optimizing the finger width and spacing, the $\mathrm{CoO}$ of the front LT-Ag can be reduced by $30 \%$ [6]. This can be further reduced up to $50 \%$ increasing the number of vias [6].

In order to show the potentiality of the MWT-SHJ architecture, $\mathrm{Cu}$ plating has been applied to this device structure. The front metallization pattern has been improved using a radial pattern. This has allowed to increase the $\mathrm{J}_{\mathrm{sc}}$ and $\mathrm{V}_{\text {oc }}$, while keeping similar FF. The IV-curve is shown in Figure 2.

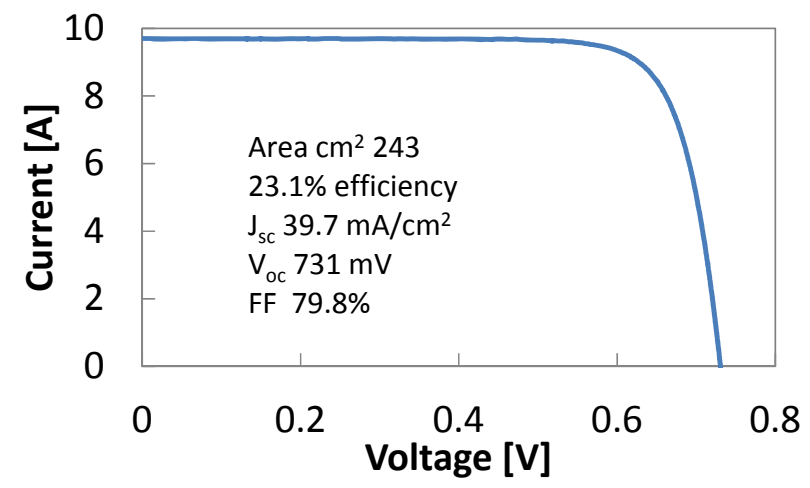

Figure 2. IV curve best cell with front cupper plating measured at ECN according to the ASTM-E948 norm using a Wacom class AAA solar simulator and spectral mismatch correction. Total area efficiency.

In our MWT-SHJ, we demonstrate $\mathrm{J}_{\mathrm{sc}}$ superior of conventional H-pattern SHJ thanks to the reduced metal front coverage as result of the carrier transport transferred to the rear side. In addition the FF increases on cell level since the current transport is not limited by the busbar width and by their number. The effect on the FF is more evident on module level. In fact the measurement setups for H-pattern and MWTSHJ solar cells are different. For the H-pattern multi-probes are usually used for the front contact of the cells. These increase the conductivity of the busbar independently of their width and thickness which results in higher FF (see table I Hpattern ref versus $4 \times 4$ ). This is not the case in real conditions (interconnection within the module) since the tabs used for the interconnection have a more limited conductivity than the multi-probes. MWT-SHJ measurements chucks do not use multi-probes as the current is transported to the rear side only thanks to the front metallization. The result is that the measurement is carried out in real module conditions.
Therefore a real comparison of the performance of MWT-SHJ and conventional front and rear contact architectures should be carried out on module level where absence of cell to module loss will be evident.

\section{A. Reverse characteristics}

MWT-SHJ $\mathrm{I}_{\text {rev }}$ current are less than $0.5 \mathrm{~A}$ at $-10 \mathrm{~V}$ for both $4 \times 4$ and $6 \times 6$ configuration (see Fig. 3) similarly to convention H-pattern SHJ.

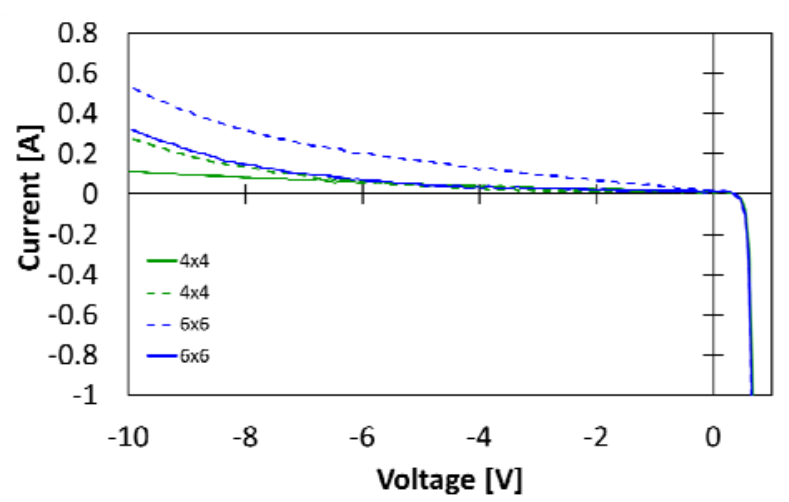

Figure 3. Reverse characteristic of MWT-SHJ solar cells with Ag screen printing.

\section{B. Sensitivity to illumination}

In order to study the behavior of the MWT-SHJ solar cells in condition of low illumination we measured their power at different illumination levels. We measured both heterojunction H-pattern and MWT-SHJ solar cells as shown in Figure 4.

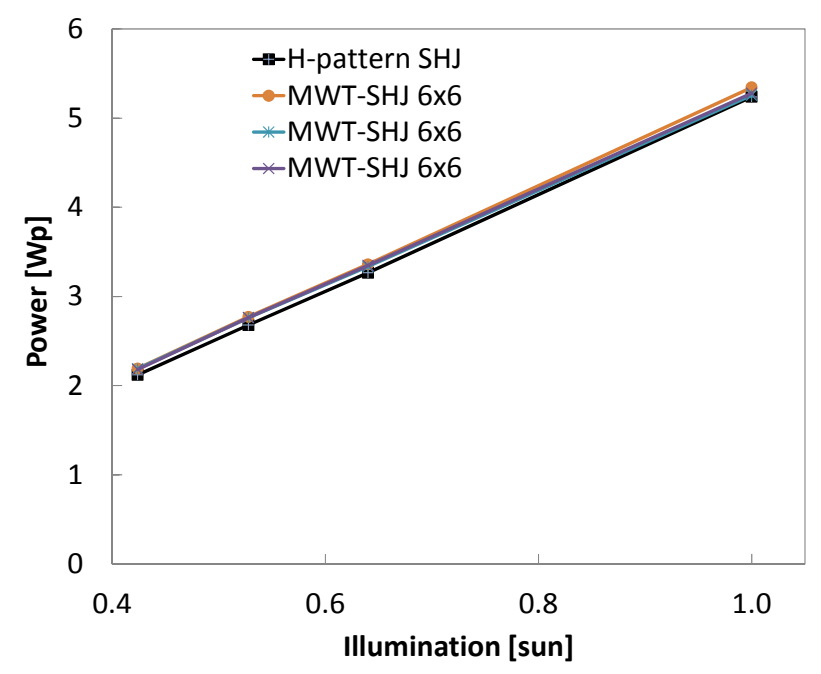

Figure 4. Efficiency versus illumination. 
No particular difference in the illumination response for MWT-SHJ solar cells with respect to conventional H-pattern silicon heterojunction solar cells. This indicates that shunt resistance impact, if any, in MWT-SHJ is comparable to that of conventional front and rear contacts H-pattern heterojunction devices.

\section{CONCLUSIONS}

We demonstrated MWT-SHJ solar cells with record efficiencies of $23.1 \%$ and $22.6 \%$ fabricated with copper plating and low temperature Ag paste, respectively. In the current configuration with LT-Ag paste and $6 \times 6$ vias we estimated a $30 \% \mathrm{CoO}$ reduction on $\mathrm{Ag}$ consumption with options to further reduce it to $50 \%$ with respect to $4 \times 4$ vias with no-extra manufacturing complexity. The two metallization concepts are not yet fully optimized and there is further room for efficiency gain. Solar cell behavior is comparable to conventional $\mathrm{HJ}$ at reverse bias voltage and low illumination intensity.

\section{REFERENCES}

[1] N. Guillevin et al, "High Power n-Type Metal-Wrap-through Cells and Modules Using Industrial Processes”, 28th European
Photovoltaic Solar Energy Conference and Exhibition, 13041310, 2013.

[2] Mikio Taguchi, Ayumu Yano, Satoshi Tohoda, Kenta Matsuyama, Yuya Nakamura, "24.7\% Record efficiency HIT solar cell on thin silicon wafer", 39th IEEE PVSC, 96-99, 2013.

[3] K. Masuko, M. Shigematsu, T. Hashiguchi, D. Fujishima, M. Kai, N. Yoshimura, T. Yamaguchi, Y. Ichihashi, T. Yamanishi, T. Takahama, M. Taguchi, E. Maruyama, S. Okamoto, "Achievement of more than 25\% conversion efficiency with crystalline silicon heterojunction solar cell”, Photovoltaic Specialists Conference (PVSC), 2014 40th IEEE, in press, 8-13 June 2014.

[4] S. Braun, G. Micard, and G. Hahn, "Solar cell improvement by using a multi-busbar design as front electrode," Energy Procedia, vol. 27, pp. 227-233, 2012.

[5] A. Schneider, L. Rubin, and G. Rubin, "Solar cell improvement by new metallization techniques-The DAY4TM electrode concept," in Proc. 4th World Conf. Photovoltaic Energy Conversion, 2006, pp. 1095-1098.

[6] G. Coletti, Y. Wu, G.Janssen, J. Loffler, B.B. van Aken, F. Li, Y. Shen, W. Yang, J. Shi, G. Li, Z. Hu, "20.3\% MWT silicon heterojunction solar cell- A novel heterojunction integrated concept embedding low Ag consumption and high module efficiency”, IEEE Journal of Photovoltaics 2014, 5, 55-60.

[7] F. Ishimura, L. Wenjun, E. Kobayashi, K. Hashimoto, S. Sato \& Y. Watabe, E. Bende, G. Coletti, "Metal Wrap through Heterojunction Solar Cell with Plated Electrode", Proceedings of the European Photovoltaic Solar Energy Conference 2016, 2016. 